\title{
RESEARCH
}

Open Access

\section{Transcriptomic metaanalyses of autistic brains reveals shared gene expression and biological pathway abnormalities with cancer}

Jaume Forés-Martos ${ }^{1}$, Ferrán Catalá-López ${ }^{1,2,3,4}$, Jon Sánchez-Valle ${ }^{5}$, Kristina Ibáñez ${ }^{6}$, Héctor Tejero ${ }^{7}$, Helena Palma-Gudiel ${ }^{1,8}$, Joan Climent ${ }^{3,9}$, Vera Pancaldi ${ }^{5}$, Lourdes Fañanás ${ }^{1,8}$, Celso Arango ${ }^{1,10}$, Mara Parellada,10, Anaïs Baudot ${ }^{11}$, Daniel Vogt ${ }^{12}$, John L. Rubenstein ${ }^{13,14}$, Alfonso Valencia ${ }^{5,15}$ and Rafael Tabarés-Seisdedos ${ }^{1,2,3^{*}}$

\begin{abstract}
Background: Epidemiological and clinical evidence points to cancer as a comorbidity in people with autism spectrum disorders (ASD). A significant overlap of genes and biological processes between both diseases has also been reported.

Methods: Here, for the first time, we compared the gene expression profiles of ASD frontal cortex tissues and 22 cancer types obtained by differential expression meta-analysis and report gene, pathway, and drug set-based overlaps between them.

Results: Four cancer types (brain, thyroid, kidney, and pancreatic cancers) presented a significant overlap in gene expression deregulations in the same direction as ASD whereas two cancer types (lung and prostate cancers) showed differential expression profiles significantly deregulated in the opposite direction from ASD. Functional enrichment and LINCS L1000 based drug set enrichment analyses revealed the implication of several biological processes and pathways that were affected jointly in both diseases, including impairments of the immune system, and impairments in oxidative phosphorylation and ATP synthesis among others. Our data also suggest that brain and kidney cancer have patterns of transcriptomic dysregulation in the PI3K/AKT/MTOR axis that are similar to those found in ASD.

Conclusions: Comparisons of ASD and cancer differential gene expression meta-analysis results suggest that brain, kidney, thyroid, and pancreatic cancers are candidates for direct comorbid associations with ASD. On the other hand, lung and prostate cancers are candidates for inverse comorbid associations with ASD. Joint perturbations in a set of specific biological processes underlie these associations which include several pathways previously implicated in both cancer and ASD encompassing immune system alterations, impairments of energy metabolism, cell cycle, and signaling through PI3K and G protein-coupled receptors among others. These findings could help to explain epidemiological observations pointing towards direct and inverse comorbid associations between ASD and specific cancer types and depict a complex scenario regarding the molecular patterns of association between ASD and cancer.
\end{abstract}

Keywords: Autism, ASD, Cancer, Transcriptome, Meta-analysis, Comorbidity, Multimorbidity, Gene expression

\footnotetext{
*Correspondence: rafael.tabares@uv.es

'Biomedical Research Networking Center of Mental Health (CIBERSAM),

Madrid, Spain

${ }^{2}$ Teaching Unit of Psychiatry and Psychological Medicine, Department of

Medicine, University of Valencia, Blasco-lbañez 15, 46010 Valencia, Spain

Full list of author information is available at the end of the article
}

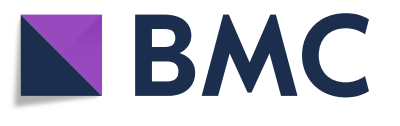

(c) The Author(s). 2019 Open Access This article is distributed under the terms of the Creative Commons Attribution 4.0 International License (http://creativecommons.org/licenses/by/4.0/), which permits unrestricted use, distribution, and reproduction in any medium, provided you give appropriate credit to the original author(s) and the source, provide a link to the Creative Commons license, and indicate if changes were made. The Creative Commons Public Domain Dedication waiver (http://creativecommons.org/publicdomain/zero/1.0/) applies to the data made available in this article, unless otherwise stated. 


\section{Background}

As Jane Austin once wrote, It is a truth universally acknowledged that [1] autism spectrum disorder (ASD) is a chronic childhood-onset neurodevelopmental condition characterized by persistent deficits in social communication and social interactions, as well as, by restricted, repetitive patterns of behavior, interests, or activities [2,3]. However, other serious clinical aspects of ASD are less well known. For instance, an increase in premature mortality has been recently reported [4-7]. ASD is among the top ten causes of disability worldwide in children between 5 and 9 years old [8], these findings could be partially explained by the link between ASD and other lifetime health problems, including epilepsy, diabetes, cardiovascular and gastrointestinal diseases, cancer, depression, and suicide [8-12]. A better understanding of these lifetime co-occurring conditions is important for people with ASD, their families and caregivers, clinicians and other healthcare professionals, scientists, and policy makers $[13,14]$. Recognizing this multimorbidity scenario, we focus our attention on the relationships between ASD and cancer for two reasons. First, evidence pointing towards different cancer rates in patients with central nervous system disorders has started to been gathered [15]. Although several studies have failed to find specific associations between ASD and cancer [16-18], others, including a large population cohort study in Taiwan [11] suggested a higher-than-expected occurrence of overall cancer in ASD patients. These authors found a standardized incidence ratio of 1.94 (95\% CI 1.18-2.99), with further increased incidence for brain and genitourinary cancers. Similarly, a large population-based case-control study in Sweden noted a significant increase in cancer mortality for all cancers combined $(\mathrm{OR}=1.80,95 \% \mathrm{CI} 1.46-2.23)$ among individuals with ASD as compared with the general population [6].In addition, mothers of children with ASD have been shown to be approximately $50 \%$ more likely to die from cancer than those of non-autistic offspring [17]. Conversely, two studies found a lower-than-expected risk of neoplasm in ASD patients, a situation that could be described as "inverse cancer comorbidity" [12, 19]. Second, given the prevalence and social impact of both diseases, further characterization of the genetic, molecular and cellular factors involved in ASD and cancer, which represent their underlying mechanisms and are used in their identification, are important and incompletely resolved issues. Recent genome-wide exome-sequencing studies of de novo variants and recurrent copy number variations (CNVs) in ASD and cancer have revealed extensive overlap in risk genes for autism and cancer [20-24]. Moreover, several studies have found a striking implication of the classically cancer related PTEN pathway in ASD [22, 2527]. These findings provide persuasive evidence of a molecular link between ASD and cancer, possibly opening the door to new treatments for both conditions. For example, chemotherapeutic agents that inhibit PTEN signaling or related pathways, such as PI3K-AKT, mTOR and NF-1 (e.g., rapamycin and everolimus), are potential candidates for treating several manifestations of autism [28].

The main goal of this study is to identify molecular mechanistic connections between the two groups of complex disorders. With this aim, we conducted meta-analyses of differential RNA expression of ASD brain tissues, and compared the dysregulated RNAs and related pathways with those involved in a collection of 22 tumor types and two non-cancer control diseases. Additionally, we employed the LINCS L1000 database $[29,30]$ to detect drugs with similar or opposite gene expression signatures to those of ASD and cancer [31]. Finally, we specifically examined which elements of the PI3K-Akt-mTOR signaling axis (involving PTEN, FMR1, NF1, TSC1, and TSC2) were dysregulated jointly in ASD and cancer [32, 33].

\section{Methods \\ Data acquisition}

Using the Gene Expression Omnibus (GEO) [34] and Array Express (AE) [35] we retrieved RNA expression studies from ASD brain tissues and cancer. To apply uniform normalization methods to microarray raw data, we selected case-control datasets belonging to the most popular single channel array platforms from Agilent, Affymetrix, and Illumina. In the case of ASD, given the small number of studies available, an RNA-Seq dataset was also incorporated.

Three studies, which included ASD and control frontal cortex samples, were found and retrieved from public repositories or were obtained directly from the authors. The datasets of Chow [36] and Voineagu [37] (GSE28475 and GSE28521) were generated using the Illumina array platform HumanRef-8 v3.0, whereas Gupta's dataset [38] was generated using Illumina's HiSeq 2000 sequencing-technology (Fig. 1a). Cerebellar, temporal, and occipital cortex samples derived from the same set of patients were also available in GSE28521 and Gupta's datasets. However, we focused on frontal cortex data to avoid introducing heterogeneity into the analysis due to tissue variability and because frontal cortex data was represented by the highest number of samples. In the case of cancer datasets, we only used primary tumors and their healthy matched control tissues, and excluded other studies and samples (i.e., metastasis and cell lines).

\section{Expression data preprocessing and normalization}

Datasets generated using Affymetrix platforms were preprocessed as follows: CEL files were retrieved from GEO or AE. The R packages oligo [39] and affy [40] were used 


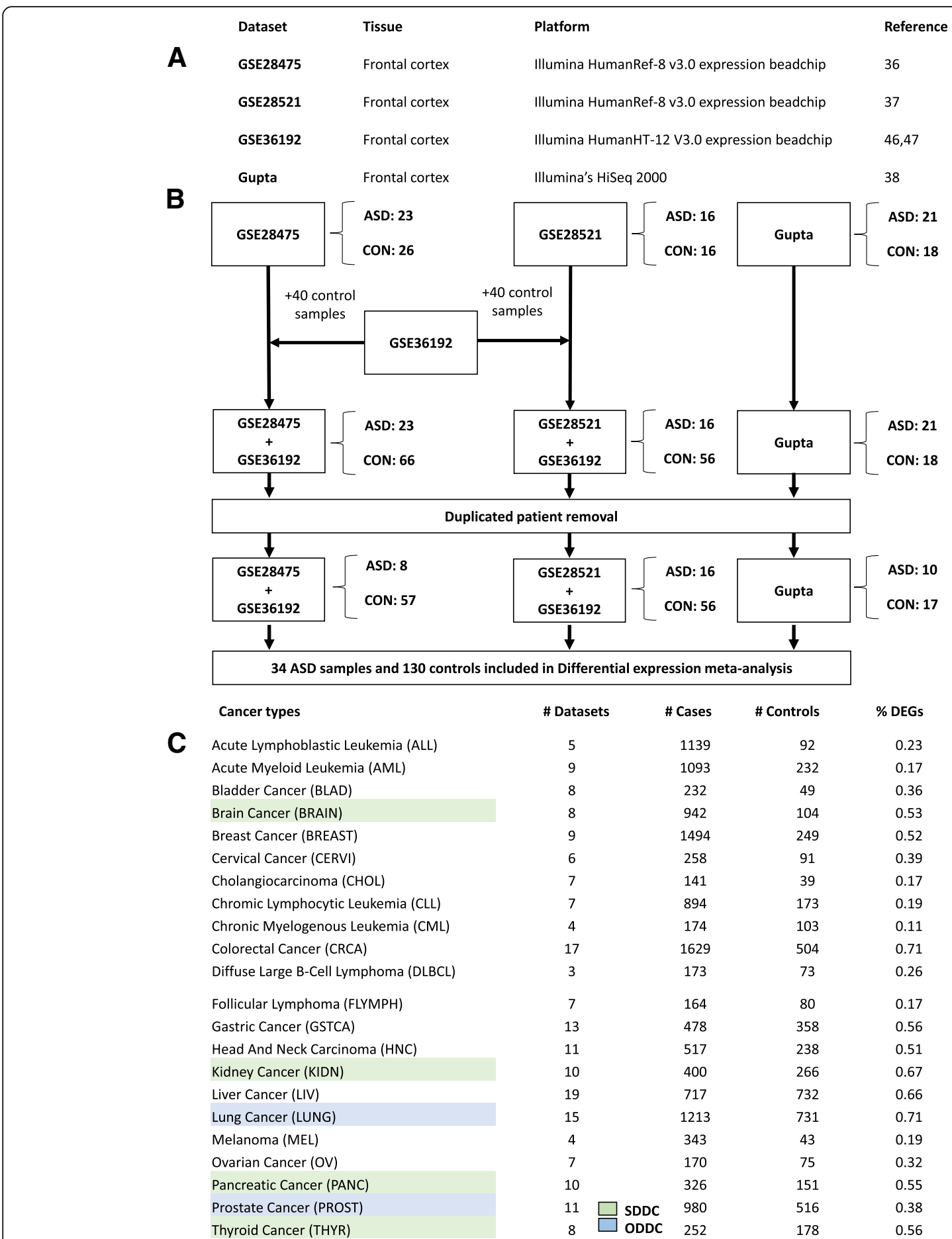

Fig. 1 a Table showing the datasets included in the ASD differential gene expression meta-analysis. $\mathbf{b}$ Diagram depicting the workflow used to perform the ASD differential gene expression meta-analysis. c Summary of the cancer types, number of datasets and samples included in each cancer-specific differential gene expression meta-analysis

to read them and to perform RMA background correction and summarization, which was followed by quantile between-sample normalization and $\log 2$ transformation. For Illumina platforms, non-normalized data were loaded to the R environment, and the Lumi [41] package was used to perform background correction using a normal exponential model fitting followed by quantile normalization and $\log 2$ transformation. Agilent data were preprocessed using the limma [42] package following the same preprocessing steps. In the case of 
RNA-Seq data, raw counts were loaded in the R environment. The Rlog function from the DESeq2 [43] package was utilized to transform the RNA-Seq count distribution to a continuous distribution suitable for integration with the array data. In short, the Rlog function transforms count data into a continuous $\log 2$ scale distribution, minimizing the differences between samples for rows with small counts and normalizing the data with respect to library size. Additional file 1: Figure S1 shows a comparison between two state of the art RNA-Seq specific differential expression methods and traditional limma analysis using Rlog transformed data suggesting that Rlog transformation renders the dataset suitable for inclusion with micro-array datasets.

To harmonize probe annotations between different dataset, platform-specific IDs were transformed into ENTREZ IDs using annotation packages. Probes targeting the same gene were collapsed using the collapseRows function from the WGCNA $[44,45]$ package selecting the MaxRowVariance method.

\section{Outlier exclusion}

Each dataset was subjected to outlier samples detection and removal using the following criteria. We computed mean inter-array correlations prior to normalization for cases and controls independently. If the mean inter-array correlation within each group was lower than 0.9 , we removed the sample showing the lowest mean inter-array correlations iteratively until a global correlation value of 0.9 was reached for both case and control groups. This method ensures that samples are not eliminated as outliers due to unbalanced case control designs while guaranteeing the elimination of samples with significant deviance from the group distributions. Additional file 1: Table S1 shows the initial number of samples included in each study and the final number of samples after exclusion criteria and outlier samples removal were performed.

\section{Addition of control samples}

The small number of samples found in the ASD brain transcriptomic studies limits the statistical power of differential expression meta-analysis. To enhance the power, searches were performed for additional datasets including frontal cortex control samples profiled with a compatible array platform. One study with such characteristics (GSE36192 [46, 47]) was found and retrieved. It included samples from the frontal lobe of the cerebral cortex profiled with Illumina HumanHT-12 V3.0. Then, we randomly included 80 frontal cortex control samples from GSE36192 to GSE28475 and GSE28475 (40 samples to each dataset) while maintaining balanced sex and age distributions between the cases and controls. No significant differences in sex, age of post mortem interval (PMI) distributions were observed between cases and controls after control sample addition (Additional file 1: Table S2). For each dataset (GSE36192 and GSE28475) data from the original study and the 40 extra control samples were merged at the raw level. Then, a normal exponential background correction method was applied to the combined data followed by quantile normalization and $\log 2$ transformation using the lumi package [41]. The combat function from the sva package [45] was finally applied to each preprocessed combination of one of the original datasets plus the control sample set in order to remove batch effects derived from different study origins (Fig. 1b).

\section{Removal of redundant patient samples}

Currently published ASD brain transcriptomic datasets rely on a set of samples derived from a partially overlapping group of patients. Eleven ASD and one control samples derived from the same patients were included in both GSE28521 and Gupta's dataset. Fourteen ASD and five control redundant samples were included in both GSE28475 and Gupta's datasets. Ten ASD and four control redundant samples respectively were shared between GSE28475 and GSE28521. Nine ASD samples derived from the same patients were present in all three datasets. No common control samples were included in the three datasets (Additional file 1: Figure S2).

Since patient redundancy could artificially inflate the number of differentially expressed genes yielded by differential gene expression meta-analysis, redundant samples were removed sequentially using the following criteria.

First, the R MetaQC package [48] was used to generate an index for the quality of each study. MetaQC integrates six quantitative quality control measures, appraising internal homogeneity of co-expression structure among studies, external consistency of co-expression patterns with a pathway database, and accuracy and consistency of differentially expressed gene detection or enriched pathway identification. For each dataset, the algorithm produces an index called standardized mean rank value (SMR) that can be interpreted as a relative measure of the quality of the study. SMR values were 1.17, 2.33 and 2.5 for GSE28521, Gupta, and GSE28475 respectively (Additional file 1: Figures S3 A and S3 B).

Additionally, as an alternative quality metric, mean inter-sample correlations were computed for each dataset. GSE28521, Gupta, and GSE28475 showed mean inter-sample correlation values of 0.96, 0.95, and 0.91, respectively, which was in agreement with the quality ordering established by MetaQC. Using both criteria, we defined GSE28521 as the highest quality study, followed by Gupta, and GSE28475. To preserve the maximum number of samples in the highest quality studies, we kept all samples in the study that showed the lowest 
SMR value and the highest mean inter-sample correlation value (highest quality). Next, the samples derived from the same patients present in both the highest quality study and the study showing the next lowest SMR and the next highest mean inter-sample correlation (the second highest quality study) were removed from the second study. Finally, from the study showing the highest SMR value and the lowest mean inter-sample correlation (lowest quality), we removed the samples derived from individuals present either in the first or in the second highest quality studies (Fig. 1b).

After control sample addition and removal of duplicated individual samples, 34 non redundant ASD cases and 130 control samples distributed in the three datasets were available to perform differential gene expression meta-analysis. No significant differences in age, sex or PMI interval composition were found either between the cases and controls ( $p$-value $>0.05$ ) (Fig. 1b). Additional file 1: Table S3 shows the samples included in the final ASD analysis and their associated covariates.

\section{Cancer and control diseases datasets}

A total of 198 datasets from 22 different cancer types comprising 18,736 samples, 13,687 tumors and 5009 tissue-matched control samples were included in our cancer differential gene expression meta-analyses (Fig. 1c, Additional file 1: Table S1). The number of included datasets for each cancer type ranged from 3 , in the case of diffuse large b-cell lymphoma, to 19 in the case of liver cancer. The sample sizes ranged from 180 in the case of cholangiocarcinoma to 2133 in the case of colorectal cancer. Malaria and ulcerative colitis were included as control diseases in order to evaluate the specificity of the associations between ASD and cancer. Ten ulcerative colitis datasets including 442 cases and 189 controls and three malaria datasets including 174 cases and 95 controls were used to perform differential gene expression meta-analyses.

\section{Differential gene expression meta-analyses}

Differential gene expression meta-analyses are known to increase the statistical power and reduce the noise of gene expression measurements [49]. For each disease, meta-analyses were carried out independently using the approach developed by Choi et al. [50] implemented in the MetaDE package [51]. All meta-analyses were performed using random effect models, since moderate to high heterogeneity was expected given the biological and technical variability present in our data. The threshold of significance was set to a conventional level of 0.05 . Thus, genes with a false discovery rate (FDR)-corrected $p$-value lower than 0.05 were considered differentially expressed.

\section{Comparison of differentially expressed gene profiles in ASD and cancer}

The expression profiles of ASD and all studied cancer types were compared to evaluate the significance of the overlaps between differentially expressed genes, as previously described [52, 53]. For each ASD-cancer pair, the significance of the four possible intersections formed by upregulated and downregulated genes was evaluated by means of one-tailed Fisher's exact tests. The intersections were:

1. Genes upregulated in both ASD and the selected cancer type (Intersection A),

2. Genes downregulated in both ASD and the selected cancer type (Intersection B),

3. Genes upregulated in ASD and downregulated in the selected cancer type (Intersection C), and

4. Genes downregulated in ASD and upregulated in the selected cancer type (Intersection D).

$P$-values were corrected by multiple testing using the FDR. Overlaps showing corrected $p$-values lower than 0.05 were considered significant. The background number of genes was set as the number of genes jointly studied in the two meta-analyses under consideration, which in turn depended on the platforms included in each meta-analysis. A cancer type was considered to be deregulated in the same direction as ASD when Intersections $\mathrm{A}$ and $\mathrm{B}$ were significant and Intersections $\mathrm{C}$ and $\mathrm{D}$ were not. These cancer types are referred to as same direction deregulated cancers (SDDCs) and could be candidates for direct comorbidity with ASD. Conversely, a cancer type was considered to be deregulated in the opposite direction from ASD when intersections $\mathrm{C}$ and $\mathrm{D}$ were significant but intersections $\mathrm{A}$ and $\mathrm{B}$ were not. These cancer types are referred to as opposite direction deregulated cancers (ODDCs) and could be candidates for inverse comorbidity with ASD.

An additional association analysis was performed on the differential expression profiles between all possible ASD and cancer pairs. Pearson's correlation coefficients of the $\hat{\mu}$ values obtained from each differential expression meta-analysis were computed. Positive correlations suggest similar patterns of differential expression while negative correlations would indicate opposite patterns.

\section{Gene set enrichment analysis}

Gene Set Enrichment Analysis (GSEA) [54] was performed in order to detect functional categories enriched in upregulated or downregulated genes. Z-values produced as output in each differential expression meta-analysis were employed as an ordering factor. For each disease, enrichment calculations were carried out using different molecular signature databases, namely, Hallmarks $(\mathrm{H})$, Canonical pathways 
(C2), and Gene Ontology (GO). A significance threshold of 0.05 was defined for the corrected $p$-value generated by the GSEA algorithm when selecting enriched functional categories.

For enrichment of gene sets placed on the intersections, a traditional overrepresentation analysis was performed using g:Profiler, an online tool for functional profiling of gene lists from large-scale experiments, through the interface $R$ package gProfileR [55].

\section{LINCS-based analysis and drug set enrichment analysis}

LINCS L1000 [27, 29, 56, 57] comprises a collection of 230,556 gene expression profiles of cancer cell lines perturbed by small molecules and genetic constructs. Here, a subset of 29,157 small molecule perturbations that was included in a custom drug classification partially based on the anatomical therapeutic chemical classification system (ATC) was selected and employed to perform drug set enrichment analyses for each studied condition as previously described in Sanchez et al. [53].

A list of genes ranked based on z-values derived from the differential gene expression analysis was generated for each disease. Then, each ranked gene list was used to compute cosine distances with each of the 29,157 perturbations included in our drug classification using the $\mathrm{R}$ ccmap package [58]. This method produces a list of perturbations or drugs ordered by its cosine distance with the target disease. Positive cosine distances indicate that a particular small molecule or drug produces a differential expression profile that mimics or resembles the differential expression profile of the disease under consideration, whereas negative cosine distances suggest that a particular small molecule or drug produces a differential expression profile that reverses the target disease profile.

Finally, the list of small molecules or drugs ordered by their cosine distances was used to detect enrichment in drug sets using a GSEA-based enrichment method implemented in the fgsea package [59]. The algorithm reveals whether a particular drug set is preferentially located at one of the extremes of the ranked list of drugs associated with each disease. Significant placement of a particular drug set at the top of the distribution suggests that it produces an effect that mimics the transcriptomic changes found in the disease under consideration. Conversely, significant placement of a particular drug set at the bottom of the perturbation distribution suggests that it produces an effect that reverses the transcriptomic changes found in the disease under consideration. A conventional FDR value of 0.05 was selected as a threshold.

We carried out this analysis for all ASD and cancer differential expression profiles. Finally, the results were compared between ASD and each tumor type.

\section{Results}

ASD differential gene-expression meta-analysis and gene set enrichment analysis (GSEA)

A total of 13,699 genes were tested for differential expression, yielding 1055 differentially expressed genes (DEGs) in ASD patients relative to controls below an FDR threshold of 0.05 . Of these DEGs, 450 were upregulated and 605 were downregulated. Full ASD differential gene expression meta-analysis results are available in Additional file 2.

Gene set enrichment analysis (GSEA) suggested that genes upregulated in ASD are mainly associated with immune system-related processes, including cytokine production, inflammatory response, leukocyte activation, NFKB signaling, interferon response and complement reaction. Cell death regulation, cell adhesion, P53 signaling, and extracellular matrix organization were also enriched in upregulated genes. Genes downregulated in ASD samples were mainly associated with oxidative phosphorylation, ATP metabolism and lactic acidosis. Neuronal system functions, such as GABA synthesis, reuptake, and degradation plus proteasome pathway related processes, were also enriched in genes downregulated in ASD samples compared to controls. Full ASD GSEA enrichment results can be found in Additional file 3.

\section{Cancer data analysis}

We found a very high proportion of differentially expressed genes in our cancer meta-analysis results, with values ranging from $11 \%$ to $71 \%$ of the total number of tested genes in chronic myeloid leukemia (CML) and colorectal cancer, respectively (Fig. 1c, Additional file 4). These proportions are compatible with previous findings for differential gene expression analysis of TCGA RNA-Seq cancer data [60], where the percentage of differentially expressed genes ranged from $32 \%$ in the case of bladder cancer to $72 \%$ in the case of breast cancer. The number of differentially expressed genes found in our analysis was correlated to the number of included studies $(r=0.76)$ and samples $(r=0.53)$, and the minimum weighted mean difference $(\hat{\mu})$ for a gene detected as significantly differentially expressed in a particular meta-analysis negatively correlated with the number of included studies $(r=-0.73)$ and samples $(r=-0.67)$. This finding indicates that as more studies were introduced in the meta-analyses, genes with smaller but consistent differences in expression were detected as significantly deregulated. The full cancer differential gene-expression meta-analyses results are available in Additional file 5.

Enrichment analysis showed that pathways associated with cell cycle such as, mitotic phase transition, DNA synthesis and repair, and telomere extension, were 
commonly upregulated in most of the cancer types (68\%). Interestingly, leukemias and lymphomas did not show changes in mitosis related pathways (Additional file 1: Figures S4 and S5). The most common downregulated pathways among cancers were related to calcium, G-protein-coupled receptors (GPCR) signaling, and fatty acid metabolism which were downregulated in between 40 and $50 \%$ of the studied cancers.

\section{Autism and cancer expression deregulation profile comparisons}

To investigate whether the transcriptomic deregulations observed in particular cancer types showed direct or inverse patterns of association with ASD, all possible ASD and cancer pairs were subjected to intersection and correlation analysis (See Methods). Four cancer types (brain, kidney, pancreatic, and thyroid cancer) presented differential gene expression profiles that were significantly deregulated in the same direction as ASD below an FDR corrected $p$-value threshold of 0.05 . These tumor types are referred to as same direction deregulated cancers (SDDC). Two tumor types (lung and prostate cancer) showed differential expression profiles deregulated in the opposite direction from frontal cortex samples of ASD patients. These types are referred to as opposite direction deregulated cancers (ODDC). No association was present between ASD and the rest of the studied cancers. (Fig. 2a).

Two hundred and fifty-four genes were found to be jointly upregulated in ASD and brain cancer and were enriched in immune system and cell death related processes. Two hundred and eighteen genes were found to be jointly downregulated in ASD and brain cancer. Enrichment in neuron and synapse related genes was found in this set of genes. Similar enrichment results were observed in the analysis of the 164 and 152 genes jointly up- and downregulated in ASD and pancreatic cancer. Kidney cancer and ASD presented 211 and 204 genes jointly up- and downregulated respectively. Shared upregulated genes between ASD and kidney cancer were also heavily enriched in immune and cell death related processes. Jointly downregulated genes in ASD and kidney cancer were enriched in mitochondrial functions and ATP synthesis. Similar results were obtained in the analysis of the 167 and 186 genes jointly up- and downregulated, respectively, in ASD and thyroid cancer, showing strong enrichment in immune system and mitochondrial function related genes in the joint upregulated and downregulated gene sets respectively.

One hundred and seventy genes were found to be jointly upregulated in ASD and downregulated in lung cancer and were enriched in immune system processes and cell death among others, whereas 229 genes were found to be downregulated in ASD and upregulated in lung cancer showing enrichment in functions related to mitochondrial function. One hundred and nineteen were found to be upregulated in ASD and downregulated in prostate cancer which were also enriched in focal adhesion, cell death and immune system processes whereas 113 genes were found to be downregulated in ASD and upregulated in prostate cancer which were enriched in mitochondrial related functions.

Additional file 1: Table S4 and Additional file 4 show the genes placed in the described intersections and the results of the overrepresentation-based functional analysis.

To determine the degree of homogeneity within SDDC and ODDC groups, we compared the content of the previously described intersections in each group. A total of $55(17 \%)$ and 24 (6\%) genes were jointly up- and downregulated respectively in the four SDDCs and ASD. Seventy (31\%) genes were upregulated in ASD and downregulated in both ODDCs, whereas 86 (35\%) were downregulated in ASD and upregulated in both ODDCs (Additional file 1: Table S5, Figure S2 B).

To evaluate the level of specificity of the reported associations between ASD and cancer and determine if associations with previous epidemiological confirmation translate in same or opposite direction deregulation patters, ASD and cancer differential expression profiles were compared to two control diseases, ulcerative colitis (UC) and malaria. Ulcerative colitis has been shown to have direct comorbid associations with both ASD and colorectal cancer (CRC) [61, 62]. UC differential expression profile was found to be deregulated in the same direction as both, ASD and colorectal cancer (Additional file 1: Figure S7 A). Nine other cancer types were found to be deregulated in the same direction than UC including all SDDCs. Prostate cancer, ALL, and CLL were found to be deregulated in opposite directions as UC. No reports investigating associations between malaria and ASD or cancer have been published to the date. Our results showed no transcriptomic associations between malaria and ASD differential expression profiles. Thyroid cancer was found to be deregulated in the same direction as malaria whereas ALL and CLL were found to be deregulated in opposite directions (Additional file 1: Figure S7 B).

Complementarily, we computed Pearson's correlation between the differential expression profiles of each possible pair of ASD and cancer to quantify the degree of association between them. SDDCs showed positive correlations with ASD. Brain cancer was the cancer type that showed the highest correlation values $(r=0.37, p<$ $0.05)$, and it was followed by kidney, thyroid and pancreatic cancer $(r=0.17, r=0.10$, and $r=0.08$, respectively, FDR < 0.05). ODDCs differential gene expression profiles presented negative correlations with ASD. Lung and 
A
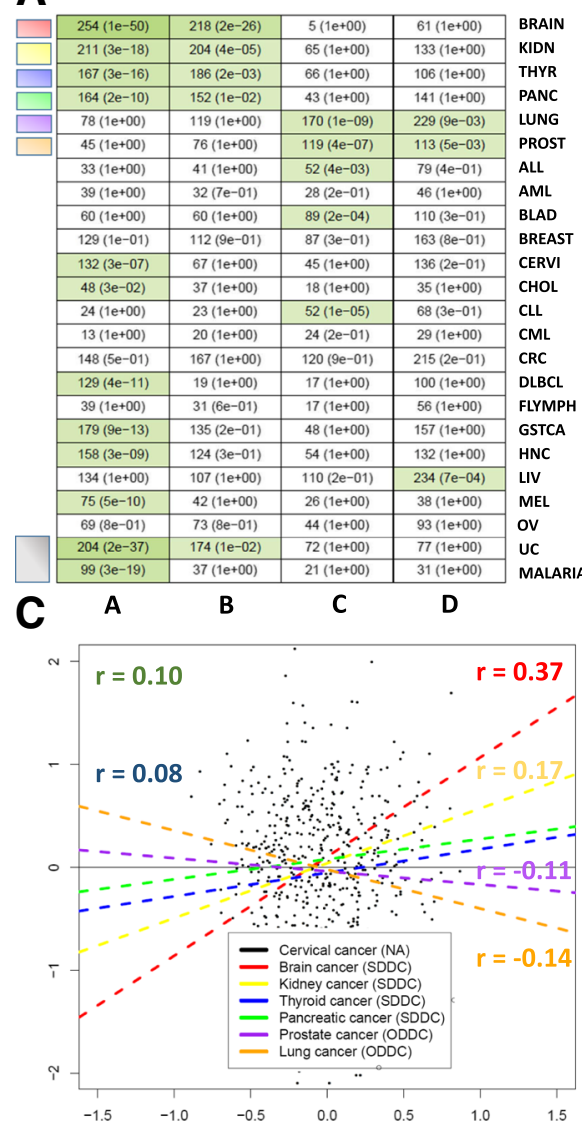

B
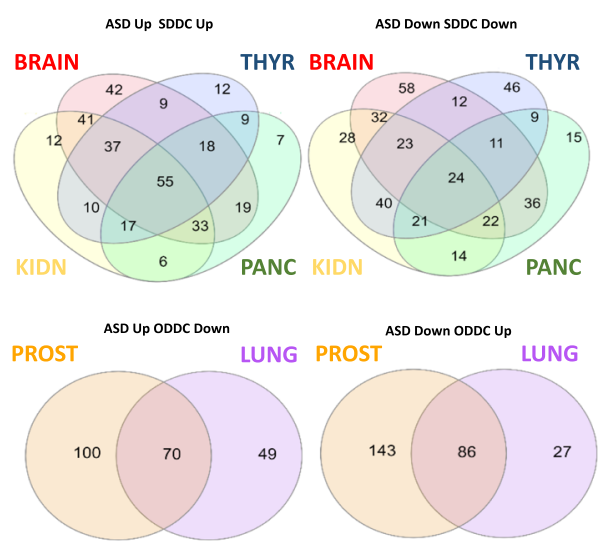

D

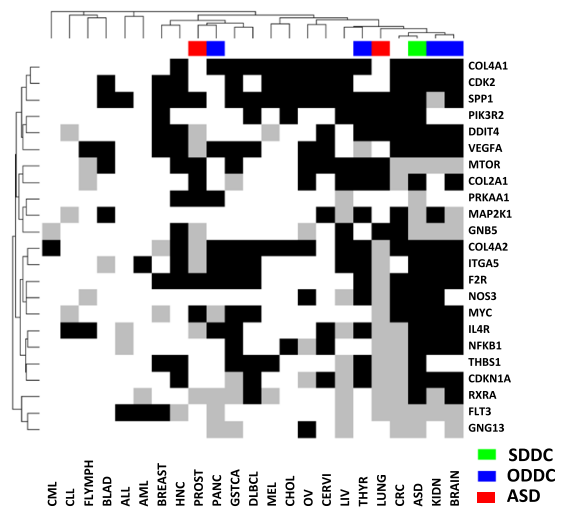

Fig. 2 a) Table showing the significance of the intersections of upregulated and downregulated genes between ASD and the 22 cancer types included in our study, comprising, acute lymphoblastic leukemia (ALL), acute myeloid leukemia (AML), bladder cancer (BLAD), brain cancer (BRAIN), breast cancer (BREAST), cervical cancer (CERVI), cholangiocarcinoma (CHOL), chronic lymphocytic leukemia (CLL), chronic myeloid leukemia (CML), colorectal cancer (CRC), diffuse large b cell lymphoma (DLBCL), follicular lymphoma (FLYMPH), gastric cancer (GSTCA), head and neck carcinoma (HNC), kidney cancer (KIDN), liver cancer (LIV), lung cancer (LUNG), melanoma (MEL), ovarian cancer (OV), pancreatic cancer (PANC), prostate cancer (PROST), and thyroid cancer (THYR). Columns A, B, C, and D include the number of genes upregulated in both, downregulated in both, upregulated in ASD and downregulated in cancer, and downregulated in ASD and upregulated in cancer, respectively. Green cell colors indicate significant intersections (FDR corrected $p$-values from Fisher's exact test lower than 0.05) with darker green tones indicating lower FDR corrected p-values. b) Venn diagrams showing the number of genes commonly deregulated in SDDCs and ODDCs. c) Scatter plots and correlation values, depicting the associations between ASD and all SDDC and ODDCs for cancer differential expression profiles. d) Heatmap showing the differential expression status of genes included in the KEGG hsa04151 pathways (PI3K-Akt signaling pathway), that were found to be differentially expressed in the ASD differential expression meta-analyses. White, gray and black cells indicate unaltered, downregulated and upregulated differential expression status, respectively

prostate cancer showed significant negative correlations $(\mathrm{r}=-0.14$ and $\mathrm{r}=-0.11$, respectively, FDR $<0.05)$. All other cancer types presented correlation absolute values lower than 0.1 (Fig. 2c). Associations showing the lowest FDR corrected $p$-values in the intersection analysis tended to present the strongest Pearson's correlations.

Partition Around Medoids (PAM) cluster analysis was carried out on the differential expression profiles of ASD and the 22 tumor types. Silhouette analysis was first applied to determine the optimum number of clusters. The five groups partition showed the highest average silhouette value suggesting that 5 was the optimum number of clusters. However, the average silhouette value was low in all cases indicating the absence of substantial structure. Results for different number of partitions can be found in Additional file 1: Figure S5. Overall, ASD tended to cluster together with brain cancer. Cancers included in the ODDC and SDDC groups tended to group together in the same cluster, indicating that their differential expression profiles were more similar between them compared to other cancer types.

A theoretical overall cancer gene expression profile was constructed by averaging the differential expression profiles of all studied cancer types. No association $(\mathrm{r}=$ 0.05) was observed between ASD and this theoretical overall cancer profiles. 


\section{$\mathrm{PI} 3 \mathrm{~K}$ associated genes}

Given the pivotal role that PI3K/AKT/MTOR plays in both ASD and cancer, we studied the differential expression status of the genes included in KEGG's hsa04151 pathway (PI3K-Akt signaling pathway). Twenty-five genes out of 272 genes belonging to hsa04151 (Fisher's exact test $\mathrm{p}$-value $=0.21$ ) were found to be deregulated in ASD below an FDR threshold of 0.05, including the core pathway gene MTOR, which was found to be downregulated in ASD. Twenty-three out of 25 genes were present in the meta-analysis of ASD and the 22 cancer types.

To determine the degree of similarity among the deregulation patterns of the 23 PI3K-associated genes observed in ASD and present in all meta-analysis cancer results, we performed hierarchical clustering using different distance measures using as an input a matrix containing discrete values for each gene representing upregulation, downregulation and normal expression status (Fig. 2d). Brain and kidney cancer clustered together with ASD with all distance measures employed suggesting common patterns of changes in this subset of genes belonging to the PI3K-Akt signaling pathway. (Additional file 1: Figure S6). In particular, F2R, MYC, NFKB1, VEGFA, DDIT4, CDKN1A, CDK2, ITGA5, COL4A1, COL4A2, and IL4R were upregulated in ASD and brain and kidney cancer, while MTOR, FLT3, and GNB5 were found to be downregulated in these three diseases.

\section{Pathway enrichment analysis comparisons and LINCs drug set analysis results}

To sketch the landscape of global common biological pathway dysregulation between ASD and cancer, we carried out functional analysis of the differential expression meta-analyses results for each included disease. To this end, GSEA and LINCS drug set enrichment analysis were performed as described in Methods. Immune system associated pathways, such as interferon alpha and gamma signaling, IL6 JAK STAT3 signaling, TNFA signaling through NFKB and MTORC1 signaling, were found to be upregulated in both ASD and 55\%, 55\%, $41 \%, 41 \%$ and $63 \%$ of cancer types, respectively. Jointly downregulated pathways between ASD and cancer were mainly associated with neuronal system genes, oxidative phosphorylation and ATP synthesis in $41 \%$ and $31 \%$ of cancers respectively. However, oxidative phosphorylation was also found to be upregulated in a subset off cancer types indicating differences in the energy metabolism abnormalities found in different tumor types. Processes downregulated in ASD and upregulated in cancer included MYC targets, DNA repair, HIV infection and proteasome activity in $77 \%, 68 \%, 55 \%$, and $46 \%$ of the studied cancers, respectively, whereas GPCR signaling and myogenesis are examples of pathways that were upregulated in ASD and downregulated in 59\% and 63\% of cancers, respectively (Fig. 3).

Drug sets commonly linked to ASD and cancer were also examined. The results suggest that treatment with mTOR inhibitors, such as everolimus, sirolimus, and temsirolimus, produce differential expression profiles that mimic the differential expression profile found in ASD while reversing the differential expression profiles found in most cancer types, excluding brain, kidney, thyroid, and pancreatic cancer, the four SDDCs.

STAT signaling inhibition by niclosamide produces differential expression profiles that mimic the ASD DEG signature while reversing the differential expression profiles of $40 \%$ of the studied cancers. Proteasome inhibitors and histone deacetylase inhibitors, such as bortozemib, entinostat and vorinostat, also mimicked ASD differential expression profile while reversing the differential expression profiles of $40 \%$ of the studied cancers (Fig. 4).

Restricting the analysis to cancers significantly associated with ASD, we observed that pathways jointly affected in ASD and SDDCs were mainly dysregulated in the same direction, i.e., they were upregulated or downregulated in both diseases. Their proportions in brain, kidney, thyroid, and pancreatic cancer were $85 \%, 89 \%$, $88 \%$ and $65 \%$, respectively, while pathways jointly affected in ODDCs and ASD were mainly deregulated in opposite directions (96\% and 95\% for lung and prostate cancer, respectively) (Additional file 1: Figure S8). Upregulated pathways shared by SDDCs and ASD were fundamentally linked to immune system-related processes. Shared downregulated pathways were implicated in oxidative phosphorylation, GPCR signaling and neuronal system genes. ODDCs upregulated pathways included cell cycle and DNA repair pathways. Contrary to what we observed in SDDCs, oxidative phosphorylation-related pathways were also upregulated in both lung and prostate cancer, indicating heterogeneity in energy metabolism abnormalities in different cancer types. Apoptotic, focal adhesion, and MAPK pathways were downregulated in ODDCs and upregulated in ASD. Finally, the MTORC1 pathway was found to be deregulated in ASD, SDDCs and ODDCs (Fig. 5).

\section{Discussion}

This is the first study aiming to explore the molecular associations between ASD and cancer at a transcriptomic level. We found positive patterns of association between ASD and four cancer types (brain, kidney, thyroid, and pancreatic) and negative patterns of association between ASD and two cancer types (lung and prostate). Brain cancer and kidney cancer showed the strongest transcriptomic associations with ASD in both intersection and correlation analyses. This observation is in 


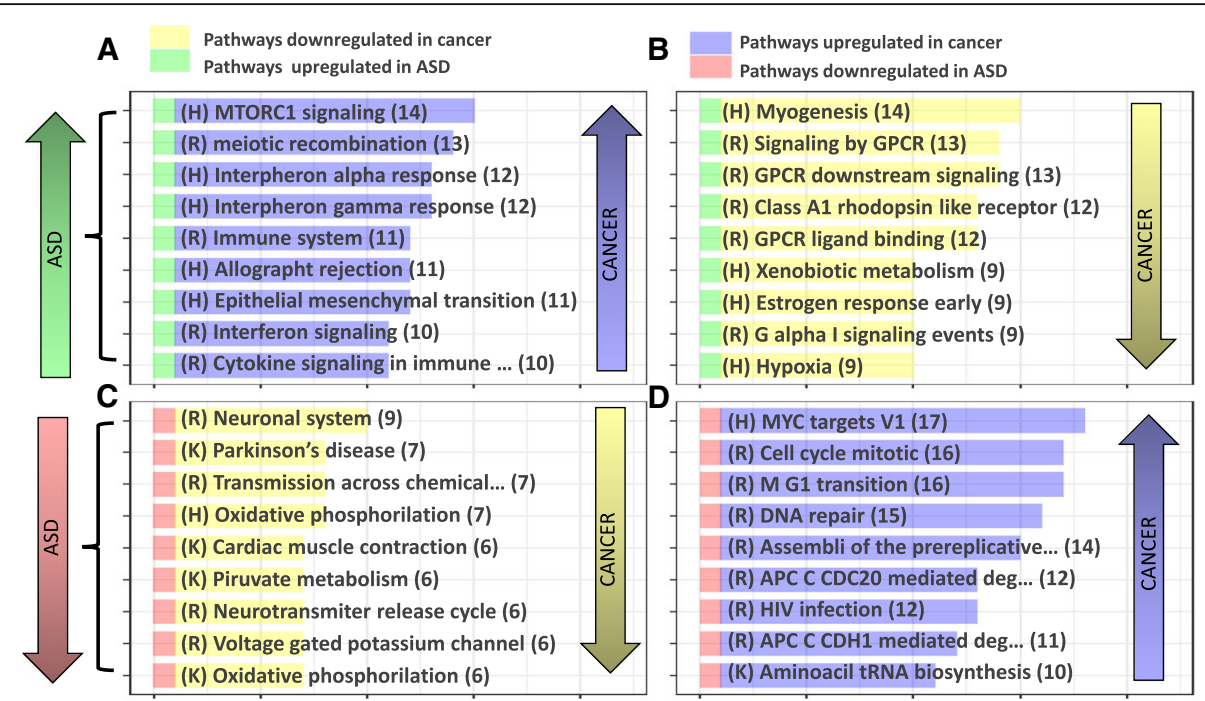

Fig. 3 Top 10 ASD- and cancer-associated pathways extracted from 3 different molecular signature databases (Hallmarks, KEGG and Reactome). Yellow and purple segments indicate pathways downregulated and upregulated in cancer, respectively, whereas red and green segments denote pathways downregulated and upregulated in ASD, respectively. The length of the yellow and purple bars indicates the number of studied cancers that represent the reported direction of deregulation for this particular pathway. a Pathways jointly upregulated in ASD and cancer. $\mathbf{b}$ Pathways upregulated in ASD and downregulated in cancer. c Pathways jointly downregulated in ASD and cancer. $\mathbf{d}$ Pathways downregulated in ASD and upregulated in cancer

agreement with previous epidemiological data reporting an increased risk of both benign and malignant brain neoplasms in patients with ASD [10]. Interestingly, the same work also noted an increased risk of congenital malformations of the urinary system in autistic individuals, including medullary sponge kidney and the presence of accessory kidneys. Epidemiological associations between urogenital system tumors and ASD have also been reported [11].

The ASD differential expression results included genes that have been previously linked to both ASD and cancer [12, 24]. For example, CUL3, a component of the multiple cullin ring ubiquitin-protein ligase complex [24], was downregulated in our ASD analysis. Furthermore, nine oncogenes present on the gene list compiled by Darbro [12] were found to be deregulated in ASD. Seven were found to be upregulated (ABL1, MYC, NFKB2, PIM1, PPARG, and BCL6) and 2 downregulated whereas two were found to be downregulated (FLT3 and MAP2K1).

On the one hand, a number of pathways were found to be commonly deregulated in different directions in ASD and several cancer types. Histone deacetylase activity, GPCR signaling, proteasome function, MYC targets, and cell cycle processes are representative examples. Some of the enumerated biological functions have previously been related to both ASD and cancer [63-68]. These abnormalities could help explain putative inverse comorbid associations between ASD and cancer.
Some biological processes were found to be deregulated in the same direction in both ASD and SDDCs, providing theoretical support for hypothetical direct comorbid associations between ASD and cancer. For instance, in agreement with previous data [69-74], our analysis suggests the presence of brain inflammation in ASD patients. Inflammatory processes are well-established drivers of carcinogenesis $[75,76]$ and are a factor that exerts direct influence on cancer-related features, such as proliferation, survival, and migration [76]. In further support of this hypothesis, indicators of ongoing inflammation were observed in several cancers, including all tumors classified as SDDCs.

Different degrees of mitochondrial activity impairment were also observed as a shared trait between ASD and SDDCs. These changes were more evident in kidney and thyroid cancers, where oxidative phosphorylation, mitochondrial electron transport chain and ATP synthesisrelated genes, including ATP50, ATP5F1, OGDHL, ATP5J, CYC1, PFKM, UQCRFS1, NDUFB6, NDUFB2, NDUFAF1, NDUFV1, DLD, and COX7B, were found to be jointly downregulated with ASD. Oxidative phosphorylation impairment, mitochondrial dysfunction and increased oxidative stress are distinctive features of autistic brains [77, 78]. Some studies have suggested that genes regulating these processes are highly enriched in parvalbumin GABAergic interneurons of the forebrain, a cell type that has been implicated in multiple murine ASD models and in humans with ASD [79]. Higher rates of 


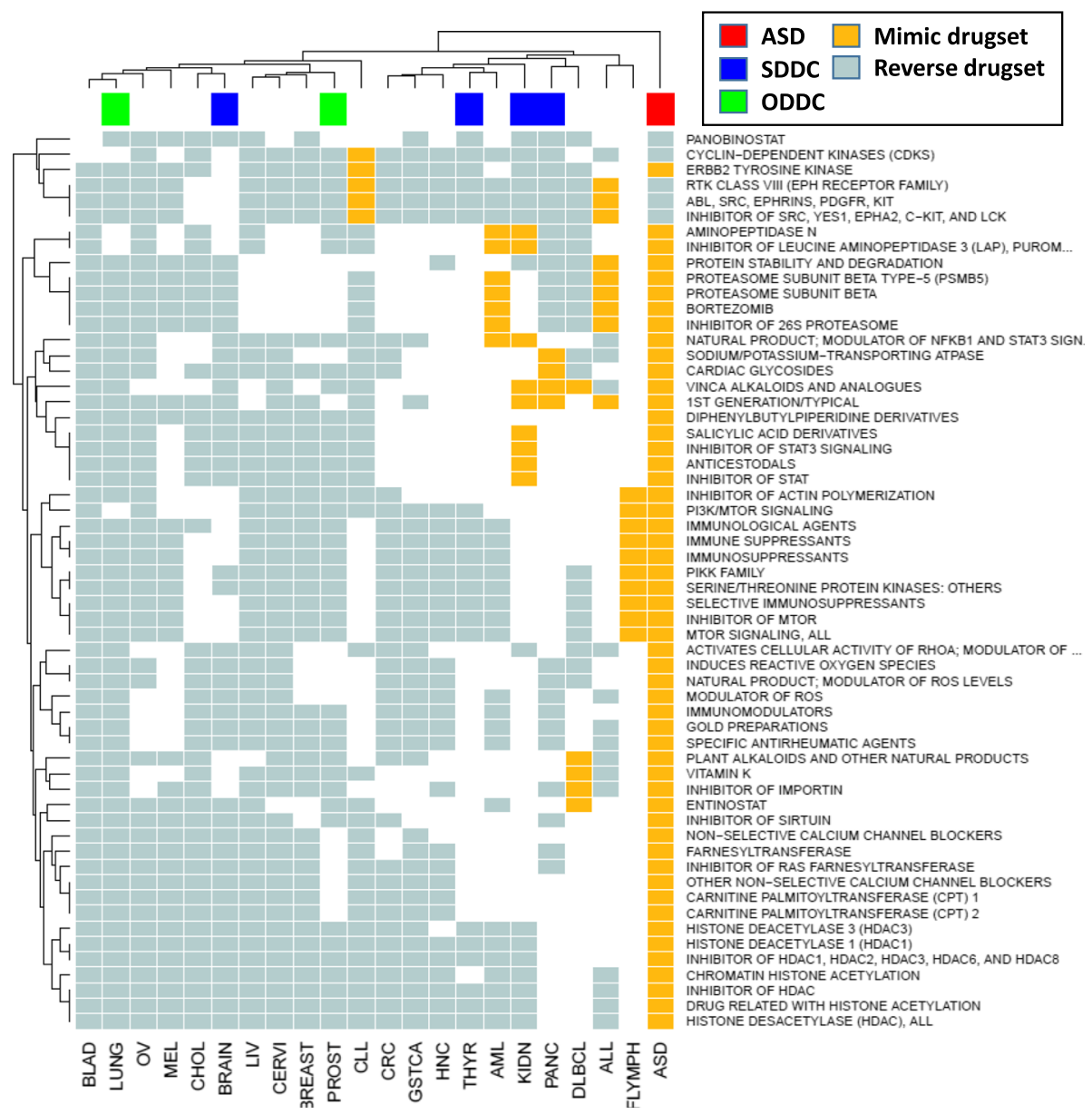

Fig. 4 LINCS L1000-derived top related drug sets. Gold cells represent drug sets that produce differential expression profiles that mimic the differential expression profiles found in the disease of interest, light blue cells indicate drug sets that generate differential expression profiles opposite to those found in our disease of interest. Green, blue and red bars located on top of the heat map indicate ODDCs, SDDCs and ASD membership, respectively

glycolysis and suppression of mitochondrial function are traits commonly observed in cancer cells. Although advances in the understanding of cancer metabolism depict oxidative phosphorylation impairment as a more complex phenomenon than previously thought [80], our data suggest that this function is commonly impaired in at least a subset of tumor types. However, some cancer types, such as lung cancer showed opposite patterns of deregulation of mitochondria and ATP synthesis related genes, highlighting the heterogeneity present in cancer energy metabolism. In addition, there is evidence indicating that inflammation and oxidative phosphorylation may have a synergic effect. Cytokines, and particularly, tumor necrosis factor alpha (TNF $\alpha$ ), impair mitochondrial oxidative phosphorylation and ATP production and increase reactive oxygen species (ROS), which in turn can increase mitochondrial injury and trigger mitochondrial content release to the cytosol, amplifying the inflammatory process [81].This interplay between the two processes may increase the risk of tumor development.

The PI3K/AKT/MTOR axis is an important target for molecular abnormalities in both ASD and cancer, which makes it a good candidate to modulate putative comorbid ASD and cancer associations. Our results showed that ASD patients presented patterns of dysregulation in this axis that are more similar to those observed in brain and kidney cancer than to any other tested cancer. Furthermore, GSEA and LINCS analyses suggested that the pathway is affected in ASD and a subset of cancers. However, given its complex nature $[82,83]$, which includes crosstalk with other signaling pathways and the presence of feedback loops, it is difficult to state whether the observed results are indicators of pathway activation or inhibition. Interestingly, ASD idiopathic cases and monogenic diseases related to autism have been linked 


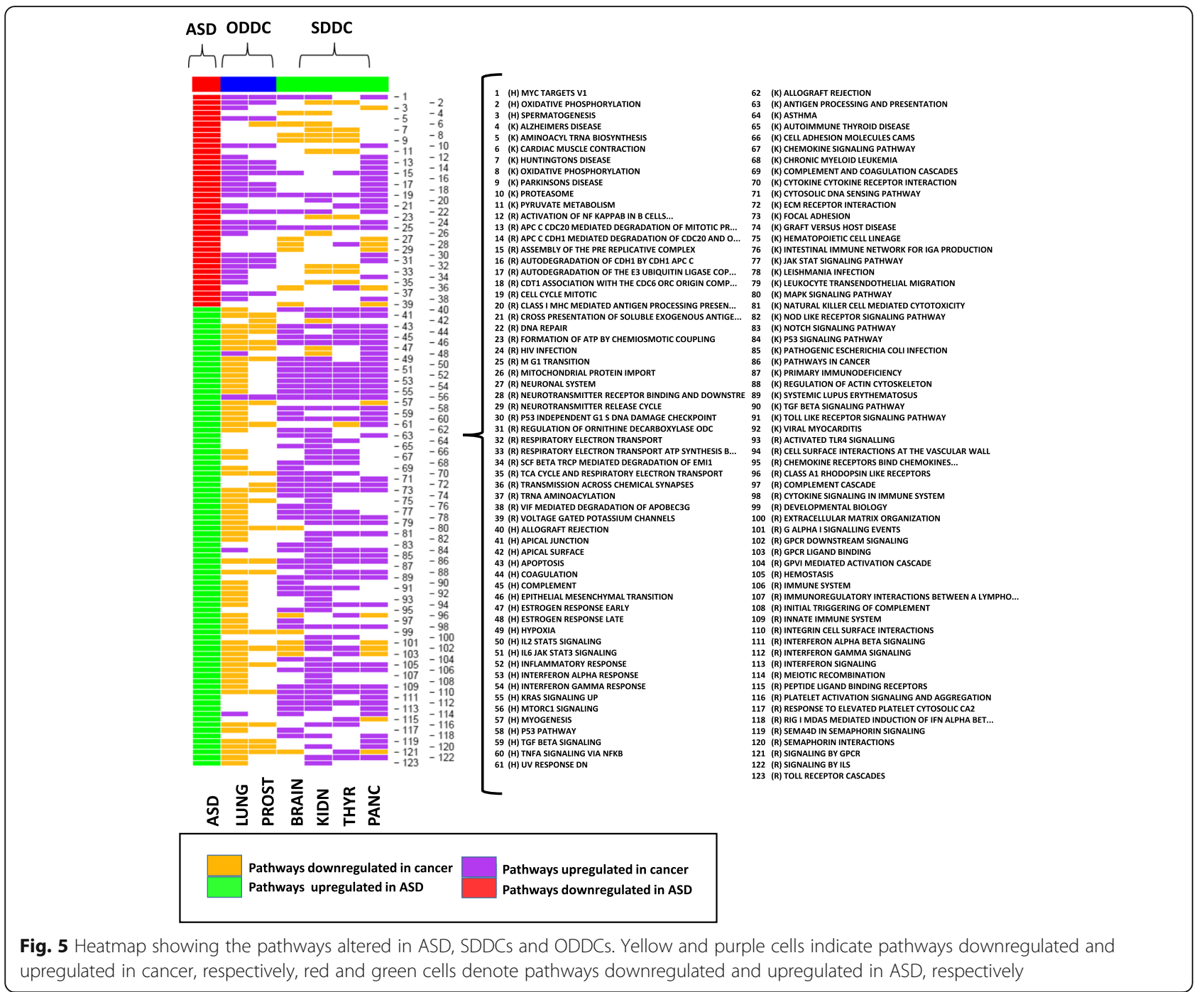

to both higher and lower activity of the PI3K/AKT/ MTOR axis [84-86]. The PI3K/AKT/MTOR axis is one the most frequently altered pathways in human tumors and directly participates in the regulation of many cancer hallmarks [87]. Moreover, it regulates several key events related to both inflammatory response, oxidative phosphorylation, and mitochondrial function [88-91]. Our observations are in agreement with a recent review highlighting the importance of the PI3K/AKT/MTOR axis and mitochondrial abnormalities as potential modulators of ASD and cancer associations [23].

The analysis regarding the two control diseases, UC and malaria, showed that previously reported direct epidemiological associations between diseases translate into similar patterns of transcriptomic deregulation between them. The increased risk of UC in ASD patients observed at a population level [61] was followed by significant same direction deregulation patterns between both diseases at a transcriptomic level. Similarly, significant same direction transcriptomic changes were observed between UC and colorectal cancer (CRC), two diseases with a known direct epidemiologic link. Furthermore, intersection analysis of UC and cancer revealed that UC was positively associated with multiple cancer types including all SDDCs and inversely associated with three cancer types including prostate cancer (ODDC). These observations indicate that the transcriptomic associations between ASD and cancer suggested by our analysis are not ASD-specific and could be shared by other diseases showing similar gene expression deregulation patterns.

Finally, we recognize some important limitations. First, despite the efforts of stablishing bio-collections aimed to uncover the genetic bases of ASD [92], the number of available datasets including gene expression data derived from autistic patient brains is scarce as it is the number of included samples in each dataset. This fact has two 
main consequences. On one hand, it undermined the statistical power of differential expression meta-analysis. Furthermore, it impedes patient stratification, which would be advisable given the intrinsic heterogeneity of ASD [93, 94]. It is reasonable to expect that different subgroups of ASD individuals present different patterns of association with cancer. We are aware that several studies, including blood-derived transcriptomic profiles of autistic patients, have been published to date; however, it still is an open question as to whether differential expression profiles derived from peripheral tissues can be used as a proxy to detect molecular abnormalities directly linked to disease physiopathology. Second, the analysis of transcriptomes is often not enough to detect whether particular biological processes were activated or inactivated, this imposes a limit to the conclusions that can be drawn from our results. Finally, although scientific evidence is starting to accumulate in favor of the presence of comorbid associations between ASD and cancer, more population and molecular studies are needed to confirm or refute competing hypotheses.

In summary, immune-related processes, mitochondrial dysfunction, and PI3K/AKT/MTOR signaling are biological processes that have been independently associated with both ASD and cancer. We have described the presence of a variable degree of changes in these pathways in SDDC, including brain and kidney cancer, the two cancer types showing the strongest associations with ASD in our intersection analysis. This observation makes these pathways good candidates to intervene to modulate putative direct comorbid associations between them. In addition, we report opposite direction associations between ASD and particular cancer types and pathways that may represent underlying molecular substrates of theoretical inverse association between ASD and cancer. These findings show a complex interplay between potential comorbid associations of ASD and cancer, and highlight the importance of further research at epidemiological, genetic, and molecular level.

\section{Conclusions}

ASD shows significant genetic and pathway deregulation overlap with several cancer types. Cancers showing positive transcriptomic patterns of association with ASD included (brain, kidney, thyroid, and pancreatic cancer) whereas cancers showing negative transcriptomic patterns of association with ASD were lung and prostate cancer. Several pathways previously associated to both diseases underlie the reported associations and include aberrant expression patterns of genes related to immune system, mitochondrial function, and PI3K/AKT/MTOR signaling among others. Further studies are needed to solidify current epidemiological and molecular knowledge about ASD and cancer multimorbidity.

\section{Additional files}

Additional file 1: Table S1. Datasets included in our study. Table S2. Age, gender and pmi distributions between cases and control samples at each step of the preprocessing procedure at the asd defferentail gene expression meta-analysis. Table S3. Final samples included in the asd meta-analysis. Table S4. Jointly same direction derregulated genes in asd ans sddcs and jointly oposite direction derregulated genes in asd and oddcs. Table S5. Genes jointly deregulated in asd and in sddcs and oddcs. Figure S1. Comaprison of differential gene expression analysis using limma with rlog transformed data and two state of the art rnaseq differential expression metdos. Figure S2. Patient overlap in the three asd studies. Figure S3. Metaqc and mean intersample correlation results. Figure S4. Partition around medoids (pam) clustering of the differential gene expression profiles of asd and the 22 included cancer types. Figure S5. Top upregulated and downregulated pathways in cancer (halmmarks molecular signature). Figure S6. Top upregulated and downretualted pathways in cancer (c2 canonical pathways molecular signature). Figure S7. Intersection analysis of the two control diseases (ulcerative colitis and malaria). Figure S8. Percentaje of pathways deregulated in the same or opposite directions between asd and cancer (DOCX $3835 \mathrm{~kb}$ )

Additional file 2: Full ASD differential gene expression meta-analysis results. (CSV $1387 \mathrm{~kb}$ )

Additional file 3: Full ASD GSEA pre-ranked enrichment results for different molecular signatures (C2, H, GO_BP, GO_CC, and GO_MF). (XLSX $698 \mathrm{~kb})$

Additional file 4: gProfileR biological process overrepresentation results of the genes contained in the significant intersections of ASD, SDDCs, and ODDCs. (XLSX $233 \mathrm{~kb}$ )

Additional file 5: Full differential gene expression meta-analysis results of cancer data. (ZIP $31237 \mathrm{~kb}$ )

\section{Abbreviations}

AE: Array express; ALL: Acute lymphoblastic leukemia; AML: Acute myeloid leukemia; ASD: Autism spectrum disorders; BLAD: Bladder cancer; BRAIN: Brain cancer; BREAST: Breast cancer; CERVI: Cervical cancer; CHOL: Cholangiocarcinoma; CLL: Chronic lymphocytic leukemia; CML: Chronic myeloid leukemia; CRC: Colorectal cancer; DLBCL: Diffuse large b cell lymphoma; FLYMPH: Follicular lymphoma; GEO: Gene expression omnibus; GSEA: Gene Set Enrichment Analysis; GSTCA: Gastric cancer; HNC: Head and neck carcinoma; KIDN: Kidney cancer; LIV: Liver cancer; LUNG: Lung cancer; MEL: Melanoma; ODDC: Opposite direction deregulated cancers; OV: Ovarian cancer; PANC: Pancreatic cancer; PROST: Prostate cancer; SDDC: Same direction deregulated cancers; THYR: Thyroid cancer

\section{Acknowledgements}

Professor Rafael Tabarés-Seisdedos and Jaume Forés were supported in part by grant number PROMETEOII/2015/021 from Generalitat Valenciana and the national grant PI17/00719 from ISCIII-FEDER.

\section{Funding}

The research was supported part by grant number PROMETEOII/2015/021 from Generalitat Valenciana and the national grant PI17/00719 from ISCIII-FEDER.

\section{Availability of data and materials}

The datasets analyzed during the current study are available in the omics data repositories stated in the main text and the Supplementary Figures and Tables file data under the reported accession numbers.

\section{Authors' contributions}

RTS conceived the study and oversaw all data collection, analyses, and writing of the manuscript. JFM carried out data collection, analysis and wrote the manuscript. JSV, KI, HT, HPG, JC, FC, and VP assisted with data acquisition and analysis. LF, CA, MP, AB, DV, JLR, and AV assisted with data interpretation and manuscript preparation. All authors read and approved the final manuscript.

Ethics approval and consent to participate Not applicable. 


\section{Consent for publication}

Not applicable.

\section{Competing interests}

The authors declare that they have no competing interests.

\section{Publisher's Note}

Springer Nature remains neutral with regard to jurisdictional claims in published maps and institutional affiliations.

\section{Author details}

'Biomedical Research Networking Center of Mental Health (CIBERSAM), Madrid, Spain. ${ }^{2}$ Teaching Unit of Psychiatry and Psychological Medicine, Department of Medicine, University of Valencia, Blasco-Ibañez 15, 46010 Valencia, Spain. ${ }^{3}$ INCLIVA Health Research Institute, Valencia, Spain. ${ }^{4}$ Department of Health Planning and Economics, National School of Public Health/IMIENS, Institute of Health Carlos III, Madrid, Spain. ${ }^{5}$ Barcelona Supercomputing Center (BSC), Barcelona, Spain. ${ }^{6}$ Genomics England, London, UK. ${ }^{7}$ Structural Biology Program, Spanish National Cancer Research Program (CNIO), Madrid, Spain. ${ }^{8}$ Anthropology Section, Department of Evolutionary Biology, Ecology and Environmental Sciences, Biomedicine Institute (IBUB), University of Barcelona (UB), Barcelona, Spain. ${ }^{9}$ Departamento de Ciencias Biomédicas, Facultad de Ciencias de la Salud, Universidad Cardenal Herrera-CEU, CEU Universities, Calle Ramon y Cajal s/n 46115 Alfara del Patriarca, Valencia, Spain. ${ }^{10}$ Department of Child and Adolescent Psychiatry, Hospital General Universitario Gregorio Marañón, liSGM, School of Medicine, Universidad Complutense, Madrid, Spain. ${ }^{11}$ Aix-Marseille Univ, Inserm, MMG, Marseille Medical Genetics, Marseille, France. ${ }^{12}$ Department of Pediatrics and Human Development, Michigan State University, East Lansing, Ml 48824, USA. ${ }^{13}$ Nina Ireland Laboratory of Developmental Neurobiology, University of California, San Francisco, CA 94158, USA. ${ }^{14}$ Department of Psychiatry, University of California, San Francisco, CA 94158, USA. ${ }^{15}$ Catalan Institution for Research and Advanced Studies (ICREA), Barcelona, Spain.

\section{Received: 17 October 2018 Accepted: 19 February 2019} Published online: 08 April 2019

\section{References}

1. Austen J. "It is a truth universally acknowledged, that a single man in possession of a good fortune must be in want of a wife." Famous first line of Pride and Prejudice (1813), by Jane Austen. Phyllis Ferguson Bottomer, author of So Odd a Mixture: Along the Autistic Spectrum in 'Pride and Prejudice' (2007), found many autistic traits in the Bennet and Fitzwilliams families. Austen wrote about them, without knowing what it was that she was describing.

2. Constantino JN, Charman T. Diagnosis of autism spectrum disorder: reconciling the syndrome, its diverse origins, and variation in expression. Lancet Neurol. 2016;15(3):279-91.

3. Association AP. Diagnostic and statistical manual of mental disorders. 5th ed; 2013.

4. Woolfenden S, Sarkozy V, Ridley G, Coory M, Williams K. A systematic review of two outcomes in autism spectrum disorder - epilepsy and mortality. Dev Med Child Neurol. 2012;54(4):306-12.

5. Schendel DE, Overgaard M, Christensen J, Hjort L, Jorgensen M, Vestergaard $M$, et al. Association of Psychiatric and Neurologic Comorbidity with Mortality among Persons with Autism Spectrum Disorder in a Danish population. JAMA Pediatr. 2016;170(3):243-50.

6. Hirvikoski T, Mittendorfer-Rutz E, Boman M, Larsson H, Lichtenstein P, Bolte S. Premature mortality in autism spectrum disorder. Br J Psychiatry. 2016; 208(3):232-8.

7. Gillberg C, Billstedt E, Sundh V, Gillberg IC. Mortality in autism: a prospective longitudinal community-based study. J Autism Dev Disord. 2010;40(3):352-7.

8. GBD 2016 Causes of Death Collaborators. Global, regional, and national agesex specific mortality for 264 causes of death, 1980-2016: a systematic analysis for the Global Burden of Disease Study 2016. Lancet. 2017; 390(10100):1151-210.

9. Lai MC, Lombardo MV, Baron-Cohen S. Autism. Lancet. 2014;383(9920):896910.

10. Lauritsen MB, Mors $\mathrm{O}$, Mortensen PB, Ewald H. Medical disorders among inpatients with autism in Denmark according to ICD-8: a nationwide register-based study. J Autism Dev Disord. 2002;32(2):115-9.
11. Chiang HL, Liu CJ, Hu YW, Chen SC, Hu LY, Shen CC, et al. Risk of cancer in children, adolescents, and young adults with autistic disorder. J Pediatr. 2015;166(2):418-23.e1.

12. Darbro BW, Singh R, Zimmerman MB, Mahajan VB, Bassuk AG. Autism linked to increased oncogene mutations but decreased Cancer rate. PLoS One. 2016;11(3):e0149041.

13. Catala-Lopez F, Hutton B, Page MJ, Ridao M, Driver JA, Alonso-Arroyo A, et al. Risk of mortality among children, adolescents, and adults with autism spectrum disorder or attention deficit hyperactivity disorder and their firstdegree relatives: a protocol for a systematic review and meta-analysis of observational studies. Syst Rev. 2017;6(1):189-017-0581-9.

14. Catala-Lopez F, Hutton B, Driver JA, Page MJ, Ridao M, Valderas JM, et al. Cancer and central nervous system disorders: protocol for an umbrella review of systematic reviews and updated meta-analyses of observational studies. Syst Rev. 2017;6(1):69-017-0466-y.

15. Catala-Lopez F, Suarez-Pinilla M, Suarez-Pinilla P, Valderas JM, GomezBeneyto M, Martinez S, et al. Inverse and direct cancer comorbidity in people with central nervous system disorders: a meta-analysis of cancer incidence in 577,013 participants of 50 observational studies. Psychother Psychosom. 2014;83(2):89-105.

16. Mouridsen SE, Rich B, Isager T. Risk of cancer in adult people diagnosed with infantile autism in childhood: A longitudinal case control study based on hospital discharge diagnoses. Research in Autism Spectrum Disorders. 2016;23:203-9.

17. Fairthorne J, Hammond G, Bourke J, Jacoby P, Leonard H. Early mortality and primary causes of death in mothers of children with intellectual disability or autism spectrum disorder: a retrospective cohort study. PLoS One. 2014;9(12):e113430.

18. Fairthorne JC, de Klerk NH, Leonard HM, Whitehouse AJ. Mothers of children with autism have different rates of Cancer according to the presence of intellectual disability in their child. J Autism Dev Disord. 2016; 46(9):3106-14.

19. Bishop-Fitzpatrick L, Movaghar A, Greenberg JS, Page D, DaWalt LS, Brilliant $\mathrm{MH}$, et al. Using machine learning to identify patterns of lifetime health problems in decedents with autism spectrum disorder. Autism Res. 2018.

20. Oikonomakis V, Kosma K, Mitrakos A, Sofocleous C, Pervanidou P, Syrmou $A$, et al. Recurrent copy number variations as risk factors for autism spectrum disorders: analysis of the clinical implications. Clin Genet. 2016:89(6):708-18.

21. Zack TI, Schumacher SE, Carter SL, Cherniack AD, Saksena G, Tabak B, et al Pan-cancer patterns of somatic copy number alteration. Nat Genet. 2013; 45(10):1134-40

22. Tilot AK, Bebek G, Niazi F, Altemus JB, Romigh T, Frazier TW, et al. Neural transcriptome of constitutional Pten dysfunction in mice and its relevance to human idiopathic autism spectrum disorder. Mol Psychiatry. 2016;21(1): $118-25$.

23. Wen $Y$, Herbert MR. Connecting the dots: overlaps between autism and cancer suggest possible common mechanisms regarding signaling pathways related to metabolic alterations. Med Hypotheses. 2017;103:118-23.

24. Crawley JN, Heyer WD, LaSalle JM. Autism and Cancer share risk genes, pathways, and drug targets. Trends Genet. 2016;32(3):139-46.

25. Frazier TW, Embacher R, Tilot AK, Koenig K, Mester J, Eng C. Molecular and phenotypic abnormalities in individuals with germline heterozygous PTEN mutations and autism. Mol Psychiatry. 2015;20(9):1132-8.

26. Vogt D, Cho KKA, Lee AT, Sohal VS, Rubenstein JLR. The parvalbumin/ somatostatin ratio is increased in Pten mutant mice and by human PTEN ASD alleles. Cell Rep. 2015;11(6):944-56.

27. Smith $\mathbf{I N}$, Thacker $\mathrm{S}$, Jaini $\mathrm{R}$, Eng C. Dynamics and structural stability effects of germline PTEN mutations associated with cancer versus autism phenotypes. J Biomol Struct Dyn. 2018;14:1-17.

28. Kilincaslan A, Kok BE, Tekturk P, Yalcinkaya C, Ozkara C, Yapici Z. Beneficial effects of Everolimus on autism and attention-deficit/hyperactivity disorder symptoms in a Group of Patients with tuberous sclerosis complex. J Child Adolesc Psychopharmacol. 2017;27(4):383-8.

29. Subramanian A, Narayan R, Corsello SM, Peck DD, Natoli TE, Lu X, et al. A Next Generation Connectivity Map: L1000 Platform and the First 1,000,000 Profiles. Cell. 2017;171(6):1437-1452.e17.

30. Lamb J, Crawford ED, Peck D, Modell JW, Blat IC, Wrobel MJ, et al. The connectivity map: using gene-expression signatures to connect small molecules, genes, and disease. Science. 2006;313(5795):1929-35. 
31. Iorio F, Bosotti R, Scacheri E, Belcastro V, Mithbaokar P, Ferriero R, et al. Discovery of drug mode of action and drug repositioning from transcriptional responses. Proc Natl Acad Sci U S A. 2010;107(33):14621-6.

32. Chen J, Alberts I, Li X. Dysregulation of the IGF-I/PI3K/AKT/mTOR signaling pathway in autism spectrum disorders. Int J Dev Neurosci. 2014;35:35-41.

33. Martini M, De Santis MC, Braccini L, Gulluni F, Hirsch E. PI3KVAKT signaling pathway and cancer: an updated review. Ann Med. 2014;46(6):372-83.

34. Gene Expression Omnibus (GEO). Available at: https://www.ncbi.nlm.nih. gov/geo/.

35. Array Express (AE). Available at: https://www.ebi.ac.uk/arrayexpress/.

36. Chow ML, Winn ME, Li HR, April C, Wynshaw-Boris A, Fan JB, et al. Preprocessing and quality control strategies for Illumina DASL assay-based brain gene expression studies with semi-degraded samples. Front Genet. 2012;3:11.

37. Voineagu I, Wang $X$, Johnston $P$, Lowe JK, Tian $Y$, Horvath $S$, et al. Transcriptomic analysis of autistic brain reveals convergent molecular pathology. Nature. 2011;474(7351):380-4.

38. Gupta S, Ellis SE, Ashar FN, Moes A, Bader JS, Zhan J, et al. Transcriptome analysis reveals dysregulation of innate immune response genes and neuronal activity-dependent genes in autism. Nat Commun. 2014;5:5748.

39. Carvalho BS, Irizarry RA. A framework for oligonucleotide microarray preprocessing. Bioinformatics. 2010;26(19):2363-7.

40. Gautier L, Cope L, Bolstad BM, Irizarry RA. Affy---analysis of Affymetrix GeneChip data at the probe level. Bioinformatics. 2004;20(3):307-15.

41. Du, P., Kibbe, W.A., Lin, S.M. lumi: a pipeline for processing Illumina microarray. Bioinformatics 2008.

42. Ritchie ME, Phipson B, Wu D, Hu Y, Law CW, Shi W, et al. limma powers differential expression analyses for RNA-sequencing and microarray studies. Nucleic Acids Res. 2015;43(7):e47.

43. Love Ml, Huber W, Anders S. Moderated estimation of fold change and dispersion for RNA-seq data with DESeq2. Genome Biol. 2014;15(12):550.

44. Langfelder P, Horvath S. Fast R functions for robust correlations and hierarchical clustering. J Stat Softw. 2012;46(11):1-17.

45. Leek JT, Johnson WE, Parker HS, Fertig EJ, Jaffe AE, Storey JD, et al. sva: Surrogate Variable Analysis. 2017.

46. Hernandez DG, Nalls MA, Moore M, Chong S, Dillman A, Trabzuni D, et al. Integration of GWAS SNPS and tissue specific expression profiling reveal discrete eQTLs for human traits in blood and brain. Neurobiol Dis. 2012; 47(1):20-8.

47. Trabzuni D, Ramasamy A, Imran S, Walker R, Smith C, Weale ME, et al. Widespread sex differences in gene expression and splicing in the adult human brain. Nat Commun. 2013;4:2771.

48. Kang D, Tseng G. MetaQC: MetaQC: Objective Quality Control and Inclusion/ Exclusion Criteria for Genomic Meta-Analysis; 2012.

49. Sweeney $T E$, Haynes WA, Vallania F, loannidis JP, Khatri P. Methods to increase reproducibility in differential gene expression via meta-analysis. Nucleic Acids Res. 2017;45(1):e1

50. Choi JK, Yu U, Kim S, Yoo OJ. Combining multiple microarray studies and modeling interstudy variation. Bioinformatics. 2003;19(Suppl 1):i84-90.

51. Wang X, Li J, Tseng GC. MetaDE: MetaDE: Microarray meta-analysis for differentially expressed gene detection; 2012.

52. Ibanez K, Boullosa C, Tabares-Seisdedos R, Baudot A, Valencia A. Molecular evidence for the inverse comorbidity between central nervous system disorders and cancers detected by transcriptomic meta-analyses. PLoS Genet. 2014;10(2):e1004173.

53. Sanchez-Valle J, Tejero H, Ibanez K, Portero JL, Krallinger M, Al-Shahrour F, et al. A molecular hypothesis to explain direct and inverse co-morbidities between Alzheimer's disease. Glioblastoma and Lung cancer Sci Rep. 2017; 7(1):4474-017-04400-6.

54. Gene Set Enrichment Analysis (GSEA). Available at: http://software. broadinstitute.org/gsea/index.jsp.

55. Reimand J, Kolde R, Arak T. gProfileR: Interface to the 'g:Profiler' toolkit; 2018.

56. Vidovic D, Koleti A, Schurer SC. Large-scale integration of small molecule-induced genome-wide transcriptional responses, Kinomewide binding affinities and cell-growth inhibition profiles reveal global trends characterizing systems-level drug action. Front Genet. 2014;5:342.

57. Library of Integrated Network-based Cellular Signatures (LINCS). Available at: http://www.lincsproject.org/.

58. Pickering A. ccmap: Combination Connectivity Mapping; 2017.
59. Sergushichev A. An algorithm for fast preranked gene set enrichment analysis using cumulative statistic calculation. bioRxiv. 2016. https://doi.org/ 10.1101/060012

60. Peng L, Bian XW, Li DK, Xu C, Wang GM, Xia QY, et al. Large-scale RNA-Seq transcriptome analysis of 4043 cancers and 548 Normal tissue controls across 12 TCGA Cancer types. Sci Rep. 2015;5:13413.

61. Lee M, Krishnamurthy J, Susi A, Sullivan C, Gorman GH, Hisle-Gorman E, et al. Association of Autism Spectrum Disorders and Inflammatory Bowel Disease. J Autism Dev Disord. 2018;48(5):1523-9.

62. Axelrad JE, Lichtiger S, Yajnik V. Inflammatory bowel disease and cancer: the role of inflammation, immunosuppression, and cancer treatment. World J Gastroenterol. 2016;22(20):4794-801.

63. Qin L, Ma K, Wang ZJ, Hu Z, Matas E, Wei J, et al. Social deficits in Shank3deficient mouse models of autism are rescued by histone deacetylase (HDAC) inhibition. Nat Neurosci. 2018;21(4):564-75.

64. Kataoka S, Takuma K, Hara Y, Maeda Y, Ago Y, Matsuda T. Autism-like behaviours with transient histone hyperacetylation in mice treated prenatally with valproic acid. Int J Neuropsychopharmacol. 2013;16(1):91-103.

65. Glozak MA, Seto E. Histone deacetylases and cancer. Oncogene. 2007;26(37): 5420-32.

66. Li Z, Zhu WG. Targeting histone deacetylases for cancer therapy: from molecular mechanisms to clinical implications. Int J Biol Sci. 2014;10(7):757-70.

67. Zheng Q, Huang T, Zhang L, Zhou Y, Luo H, Xu H, et al. Dysregulation of ubiquitin-proteasome system in neurodegenerative diseases. Front Aging Neurosci. 2016;8:303.

68. Manasanch EE, Orlowski RZ. Proteasome inhibitors in cancer therapy. Nat Rev Clin Oncol. 2017;14(7):417-33.

69. Kern JK, Geier DA, Sykes LK, Geier MR. Relevance of Neuroinflammation and encephalitis in autism. Front Cell Neurosci. 2016;9:519.

70. Pardo CA, Vargas DL, Zimmerman AW. Immunity, neuroglia and neuroinflammation in autism. Int Rev Psychiatry. 2005;17(6):485-95.

71. Vargas DL, Nascimbene C, Krishnan C, Zimmerman AW, Pardo CA. Neuroglial activation and neuroinflammation in the brain of patients with autism. Ann Neurol. 2005;57(1):67-81.

72. Zimmerman AW, Jyonouchi H, Comi AM, Connors SL, Milstien S, Varsou A et al. Cerebrospinal fluid and serum markers of inflammation in autism. Pediatr Neurol. 2005;33(3):195-201.

73. Morgan JT, Chana G, Pardo CA, Achim C, Semendeferi K, Buckwalter J, et al. Microglial activation and increased microglial density observed in the dorsolateral prefrontal cortex in autism. Biol Psychiatry. 2010;68(4):368-76.

74. Tetreault NA, Hakeem AY, Jiang S, Williams BA, Allman E, Wold BJ, et al. Microglia in the cerebral cortex in autism. J Autism Dev Disord. 2012;42(12):2569-84.

75. Okada F. Inflammation-related carcinogenesis: current findings in epidemiological trends, causes and mechanisms. Yonago Acta Med. 2014; 57(2):65-72.

76. Coussens LM, Werb Z. Inflammation and cancer. Nature. 2002;420(6917): 860-7.

77. Rossignol DA, Frye RE. Mitochondrial dysfunction in autism spectrum disorders: a systematic review and meta-analysis. Mol Psychiatry. 2012;17(3):290-314.

78. Markkanen E, Meyer U, Dianov GL. DNA Damage and Repair in Schizophrenia and Autism: Implications for Cancer Comorbidity and Beyond. Int J Mol Sci. 2016;17(6). https://doi.org/10.3390/ijms17060856.

79. Paul A, Crow M, Raudales R, He M, Gillis J, Huang ZJ. Transcriptional Architecture of Synaptic Communication Delineates GABAergic Neuron Identity. Cell. 2017;171(3):522-539.e20

80. Ashton TM, McKenna WG, Kunz-Schughart LA, Higgins GS. Oxidative phosphorylation as an emerging target in cancer therapy. Clin Cancer Res. 2018.

81. van Horssen J, van Schaik P, Witte M. Inflammation and mitochondrial dysfunction: a vicious circle in neurodegenerative disorders? Neurosci Lett. 2017.

82. Efeyan A, Sabatini DM. mTOR and cancer: many loops in one pathway. Curr Opin Cell Biol. 2010;22(2):169-76.

83. Mendoza MC, Er EE, Blenis J. The Ras-ERK and PI3K-mTOR pathways: crosstalk and compensation. Trends Biochem Sci. 2011;36(6):320-8.

84. Onore C, Yang H, Van de Water J, Ashwood P. Dynamic Akt/mTOR signaling in children with autism Spectrum disorder. Front Pediatr. 2017:5:43.

85. Enriquez-Barreto L, Morales M. The PI3K signaling pathway as a pharmacological target in Autism related disorders and Schizophrenia. Mol Cell Ther. 2016:4:2-016-0047-9 eCollection 2016.

86. Nicolini C, Ahn Y, Michalski B, Rho JM, Fahnestock M. Decreased mTOR signaling pathway in human idiopathic autism and in rats exposed to valproic acid. Acta Neuropathol Commun. 2015;3:3-015-0184-4. 
87. Fruman DA, Rommel C. PI3K and cancer: lessons, challenges and opportunities. Nat Rev Drug Discov. 2014;13(2):140-56.

88. Hawkins PT, Stephens LR. PI3K signalling in inflammation. Biochim Biophys Acta. 2015:1851(6):882-97.

89. Stark AK, Sriskantharajah S, Hessel EM, Okkenhaug K. PI3K inhibitors in inflammation, autoimmunity and cancer. Curr Opin Pharmacol. 2015;23:82-91.

90. Cerniglia GJ, Dey S, Gallagher-Colombo SM, Daurio NA, Tuttle S, Busch TM, et al. The PI3K/Akt pathway regulates oxygen metabolism via pyruvate dehydrogenase (PDH)-Elalpha phosphorylation. Mol Cancer Ther. 2015; 14(8):1928-38.

91. Altomare DA, Khaled AR. Homeostasis and the importance for a balance between AKT/mTOR activity and intracellular signaling. Curr Med Chem. 2012;19(22):3748-62.

92. Reilly J, Gallagher L, Chen JL, Leader G, Shen S. Bio-collections in autism research. Mol Autism. 2017:8:34-017-0154-8 eCollection 2017.

93. Masi A, DeMayo MM, Glozier N, Guastella AJ. An overview of autism Spectrum disorder, Heterogeneity and Treatment Options. Neurosci Bull. 2017;33(2):183-93.

94. Brown EA, Lautz JD, Davis TR, Gniffke EP, VanSchoiack AAW, Neier SC, et al, Clustering the autisms using glutamate synapse protein interaction networks from cortical and hippocampal tissue of seven mouse models. Mol Autism. 2018:9:48-018-0229-1 eCollection 2018.

Ready to submit your research? Choose BMC and benefit from:

- fast, convenient online submission

- thorough peer review by experienced researchers in your field

- rapid publication on acceptance

- support for research data, including large and complex data types

- gold Open Access which fosters wider collaboration and increased citations

- maximum visibility for your research: over $100 \mathrm{M}$ website views per year

At $\mathrm{BMC}$, research is always in progress.

Learn more biomedcentral.com/submissions 